American J. of Engineering and Applied Sciences 4 (4): 520-530, 2011

ISSN 1941-7020

(C) 2014 X. Tang et al., This open access article is distributed under a Creative Commons Attribution

(CC-BY) 3.0 license

\title{
Coherent Polarization Shift Keying Modulated Free Space Optical Links Over a Gamma-Gamma Turbulence Channel
}

\author{
${ }^{1}$ Tang, X., ${ }^{1}$ Z. Ghassemlooy, \\ ${ }^{1}$ S. Rajbhandari, ${ }^{2}$ W.O. Popoola and ${ }^{3}$ C.G. Lee \\ ${ }^{1}$ School of Computing, Optical Communications Research Group, \\ Engineering and Information Sciences, Northumbria University, UK \\ ${ }^{2}$ Institute for Digital Communications, School of Engineering, \\ University of Edinburgh, Edinburgh, UK \\ ${ }^{3}$ Department of Electronic Engineering, Chosun University, \\ Gwangju, Republic of Korea
}

\begin{abstract}
Problem statement: The optical signal propagating through the Free Space Optical (FSO) channel suffers from irradiance and phase fluctuations caused by the atmospheric turbulence, which results in Bit Error Rate (BER) performance degradation. Approach: In this study the performance of the Multilevel Coherent Polarization Shift Keying (M-POLSK) based FSO communication system operating over the gamma-gamma turbulence channel is investigated. To mitigate the turbulence induced fading, the convolutional coding and spatial diversity techniques are employed. The upper BER bounds are derived using the transfer function technique. Results: For example, with a SNR of $30 \mathrm{~dB}$, the BERs for uncoded and coded M-POLSK are 0.047 and $1.4 \times 10^{-4}$, respectively in the weak turbulence regime. When the Maximum Ration Combining (MRC) technique employing four detectors are used in the receiver, the power gains of $\sim 31.4, \sim 29.5$ and $\sim 57.9 \mathrm{~dB}$ are achieved for weak, moderate and strong turbulence regimes, respectively. Conclusion: We have also shown that the spatial diversity offers increased link margin as the scintillation level rises. Compared to the angular modulation, the proposed M-POLSK scheme offers high immunity to the phase noise, thus reducing the power penalties.
\end{abstract}

Key words:FSO, Maximum Ration Combining (MRC), Spatial Diversity (SD), atmospheric turbulence channel, Field of View (FOV), Polarization Controller (PC), Polarization Beam Splitter (PBS), Phase Modulator (PM)

\section{INTRODUCTION}

Unlike Radio Frequency (RF) based wireless systems which suffer from bandwidth constraints, FSO systems offers full-duplex gigabit rate throughput. This makes it a suitable technology for delivering broadband wireless services for certain applications including the metropolitan area network, enterprise/local area networks, optical fiber backup, enterprise connectivity and the last mile access networks (Uysal et al., 2006). FSO offers a number of advantages over the RF technology, including higher data rate, an unregulated spectrum, high immunity to the electromagnetic interference, high security, a small size transceiver, low cost and a lower power consumption (Popoola and Ghassemlooy, 2009; Popoola et al., 2008). In indoor applications, optical radiation is confined within rooms (assuming no windows and transparent barriers), thus making eavesdropping a difficult task. For outdoor applications, a laser transmitter with a highly directional and a cone-shaped beam profile normally installed high above the street level thus makes interception difficult. Therefore, anyone trying to tap into the communication link can be easily detected and any equipment placed within the narrow optical foot print could easily be identified.

However, the FSO link performance suffers from a number of phenomena such as the misalignment due to the building sway caused by the wind, thermal expansion and weak earthquakes (Uysal et al., 2006; Arnon, 2003; Tang et al., 2011). Another important issue with the outdoor FSO system is the susceptibility of the optical link to the atmospheric conditions. The laser beam propagating through the channel suffers

\section{Corresponding Author: Tang, X., School of Computing, Optical Communications Research Group,}


from the high attenuation due to the atmospheric scatters such as haze, fog and rain, which limit the link range and the system reliability. Fog compared with rain and haze is the biggest contributor to the path loss. The attenuation due to thick fog and haze can reach up to $300 \mathrm{~dB} \mathrm{~km}^{-1}$ thus limiting the link range to $100 \mathrm{~m}$ (Ramirez-iniguez et al., 2008). Smoke also has a similar effect as fog on the propagating optical signal (Ramireziniguez et al., 2008).

Even in clear sky conditions, the optical signal suffers from the atmospheric turbulence, which is also known as the scintillation (Popoola and Ghassemlooy, 2009; Kamalakis et al., 2006). Scintillation originates from the inhomogeneities in the refraction index of the atmosphere caused by the variation in the temperature and the pressure. Scintillation leads to significant fluctuations on the amplitude and phase of the optical field (i.e., channel fading) (Popoola and Ghassemlooy, 2009). The knowledge of statistical distribution of the atmospheric turbulence is necessary to fully study and predict FSO performance operating over a clear atmospheric condition.

The performance impairments due to the scintillation can be mitigated by adopting several approaches including the aperture averaging and the diversity techniques (Uysal et al., 2006; Khalighi et al., 2009; Zhu and Kahn, 2003), the adaptive optics (Weyrauch and Vorontsov, 2004), the saturated optical amplifiers (Abtahi et al., 2006), the modulation techniques and the error control coding (Uysal et al., 2006; Zhu and Kahn, 2003). In (Uysal et al., 2006; Zhu and Kahn, 2001; 2003) the performances of coded FSO links for the log-normal and gamma-gamma channel modelsunder atmospheric turbulence have been investigated. Study also presents an approximate upper bound for the Pairwise Error Probability (PEP) and the upper bounds on the BER using the transfer function technique for the coded FSO links with Intensity Modulation/Direct Detection (IM/DD). The diversity techniques comprising space, time, or frequency (wavelength) have been adapted to improve performance impairments due to the scintillation. With the spatial diversity technique, where a single receiver with a large Field of View (FOV) is replaced by a group of detectors with a narrow FOV, the possibility of all the detectors suffering from the deep fade simultaneously is much reduced. Moreover, the spatial diversity scheme limits the amount of background light from unwanted sources that impinges on the specific detector, which otherwise could be received by the single receiver with a wide FOV (Khalighi et al., 2009).

The type of modulation schemes in FSO systems is crucial to ensure the maximum power efficiency. The coherent system based on the angular modulation; such as Binary Phase Shift Keying (BPSK) and Differential
Phase Shift Keying (DPSK) are highly sensitive to the phase noise effects (Betti et al., 1995). Alternatively, it has been shown that POLSK offers higher immunity to the phase noise and the atmospheric turbulence. The polarization states of the laser beam propagating through the FSO channel can be maintained over a long link range (Sugianto and Davis, 2006).

\section{MATERIALS AND METHODS}

The aim of this study is to evaluate the performance of the Multilevel POLSK (M-POLSK) modulated FSO system with the coherent detection operating over the gamma-gamma atmospheric turbulence channel. For this purpose, the performance improvement by the error control coding and the spatial diversity will be also considered. However, the code should be short and simple in order to keep the complexity of this approach reasonably low. The system performance will be compared with the coherent BPSK and DPSK in terms of the average BER. The noise, comprising of both the background radiation and the thermal noise, is modeled as the additive white Gaussian process. We also assume that the transmitter and the receiver have the perfect link alignment.

Gamma-gamma turbulence model: The analysis for the atmospheric turbulence has been carried out by a number of researchers and several theoretical models have been developed to characterize its behaviour. The simplest and most widely reported model is the log normal turbulence, which is mathematically convenient and tractable. The log normal model is based on the Rytov approximation, which requires the unperturbed phase gradient to be large compared to the magnitude of the scattering field wave (Zhu and Kahn, 2003; Popoola and Ghassemlooy, 2009). However, the log normal model only covers the weak turbulence regime with a single scattering event. For the turbulence in the saturation regime with multiple scatterings, the log normal model becomes invalid (Uysal et al., 2006; Osche, 2002; Karp, 1988). The strength of turbulence can be described by the $\log$ intensity variance $\sigma^{2}{ }_{1}$ and the $\log$ normal model is only valid for $\sigma_{1}^{2}$. Another important parameter for describing the turbulence strength is the scintillation index, which is the log intensity variance normalized by the square of the mean irradiance. The experimental results have indicated that the scintillation index does not only saturate, but also decreases after it researches the maximum value while the strength of turbulence continues rising (Karp, 1988). In the saturation regime and beyond with the link length of several kilometres the intensity 
fluctuation is experimentally verified to obey the Rayleigh distribution (Karp, 1988; Popoola and Ghassemlooy, 2009). To express the turbulence effects across all regimes, the gamma-gamma turbulence model is considered in study.

The gamma-gamma model first proposed by Andrews et al. (2001) is valid for all turbulence regimes from weak to strong regimes. It is based on the assumption that the fluctuation of the laser beam propagating through the turbulence medium consists of the refraction $\left(I_{1}\right)$ and scattering $\left(I_{2}\right)$ effects. Subsequently, the normalized irradiance is expressed as the normalized product of two independently statistical random processes $I_{1}$ and $I_{2}$ given by Eq. 1:

$$
\mathrm{I}=\mathrm{I}_{1} \mathrm{I}_{2}
$$

where, $I_{1}$ and $I_{2}$ represent the refraction and scattering effects, respectively and both are governed by the gamma distribution (Andrews et al., 2001; Al-Habash et al., 2001). Thus, the Probability Density Function (PDF) of the gamma-gamma model for the received irradiance fluctuation is derived as Eq. 2:

$$
\mathrm{p}(\mathrm{I})=\frac{2(\alpha \beta)^{(\alpha+\beta) / 2}}{\Gamma(\alpha) \Gamma(\beta)} \mathrm{I}^{\frac{\alpha+\beta}{2}-1} \mathrm{~K}_{\alpha-\beta}(2 \sqrt{\alpha \beta \mathrm{I}}), \mathrm{I}>0
$$

where, $\Gamma(\cdot)$ represents the gamma function and $\mathrm{K}_{\mathrm{n}}($. is the modified Bessel function of the 2nd kind of order $n$. The parameters $\alpha$ and $\beta$ characterize the optical power fluctuation PDF which are related to the atmospheric conditions. Assuming that the optical radiation at the receiver is a plane wave, $\alpha$ and $\beta$ can be expressed as Eq. 3a-b (Popoola and Ghassemlooy, 2009; Andrews et al., 2001):

$$
\begin{aligned}
& \alpha=\left\{\exp \left[\frac{0.49 \sigma_{1}^{2}}{\left(1+0.18 \mathrm{~d}^{2}+0.56 \sigma_{1}^{12 / 5}\right)^{7 / 6}}\right]-1\right\}^{-1} \\
& \beta=\left\{\exp \left[\frac{0.51 \sigma_{1}^{2}\left(1+0.69 \sigma_{1}^{12 / 5}\right)^{-5 / 6}}{\left(1+0.9 \mathrm{~d}^{2}+0.62 \mathrm{~d}^{2} \sigma_{1}^{12 / 5}\right)^{5 / 6}}\right]-1\right\}^{-1}
\end{aligned}
$$

$$
\begin{aligned}
& \text { Where } \\
& \sigma_{1}^{2}=0.5 \mathrm{C}_{\mathrm{n}}^{2} \mathrm{k}^{7 / 6} \mathrm{~L}^{11 / 6} \quad=\text { Log irradiance variance } \\
& \mathrm{d}=\left(\mathrm{kD}^{2} / 4 \mathrm{~L}\right)^{1 / 2}, \mathrm{k}=2 \pi / \lambda=\text { Optical wave number } \\
& \lambda \quad=\text { Wavelength } \\
& \mathrm{D} \\
& \text { L L } \\
& =\text { Diameter of the receiver } \\
& \text { collecting lens aperture } \\
& =\text { Link length in meters }
\end{aligned}
$$

$$
\mathrm{C}_{\mathrm{n}}^{2}=10^{-15} \mathrm{~m}^{-2 / 3}
$$

$=$ The typical average value of refractive index structure parameter

For FSO links near the ground, $\mathrm{C}_{\mathrm{n}}^{2}$ can be taken approximately as $1.7 \times 10^{-14} \mathrm{~m}^{-2 / 3}$ and $8.4 \times 10^{-15} \mathrm{~m}^{-2 / 3}$ during daytime and night (Goodman, 1985; Uysal et al., 2006).

\section{M-Polsk modulation principles:}

A. transmitter: Figure 1 illustrates the block diagram of the M-POLSK transmitter. The state of the polarization of the laser beam is controlled by a Polarization Controller (PC) before being fed into the Polarization Beam Splitter (PBS). The Transmitting Laser (TL) beam is linearly polarized and has a $\pi / 4$ polarization with respect to the principle axe of the external Phase Modulator (PM).

The emitted optical field of the carrier is expressed by a complex vector $\overrightarrow{\mathrm{E}} 0_{\mathrm{S}}(\mathrm{t})$ in the transverse plane, which is given as Eq. 4 (Cusani et al., 1992):

$$
\overrightarrow{\mathrm{E}}_{0}(\mathrm{t})=\sqrt{\frac{\mathrm{P}}{2}} \mathrm{e}^{\mathrm{j}[\omega t+\varphi(t)]}\{\hat{\mathrm{x}}+\hat{\mathrm{y}}\}
$$

where, $\mathrm{P}, \omega$ and $\varphi(t)$ are the power, the angular frequency and the phase noise of the emitted optical carrier, respectively. The two vectors $\hat{x}$ and $\hat{y}$ and denote the direction along which the field is polarized. Note that the square root of the field power directly provides the amplitude.

The signal $\overrightarrow{\mathrm{E}}_{0}(\mathrm{t})$ is decomposed by the PBS into two orthogonally polarized components with the equal amplitudes. The amplitude and phase of the optical component polarized along the $\hat{\mathrm{x}}$ axis are modulated externally by the data while the $\hat{y}$ component is transmitted as the reference carrier. The applied voltage to the $\mathrm{LiNbO}_{3}$ based external phase modulator is equal to either zero or $\mathrm{V}_{\pi}$. The applied voltage $\mathrm{V}_{\pi}$ induces $\pi$ phase shift in the $\hat{x}$-component and zero phase shift in the $\hat{y}$-component, thus leading to a $\pi / 2$ rotation of the polarization of the optical carrier. The amplitude modulation combined with the phase modulation in $\hat{x}$ component is described as an eightlevel modulation where the time axis is divided into symbol intervals. 


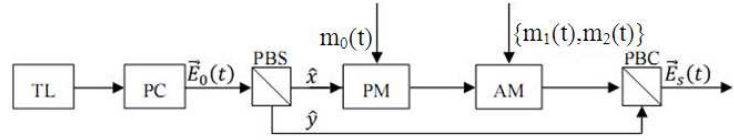

Fig 1: Block diagram of M-POLSK transmitter. AM (amplitude modulator).

Each symbol is associated to a value of the optical field and during a symbol interval the transmitted field is constant. The transitions between subsequent symbol intervals are supposed to be instantaneous. Under these conditions, the transmitted optical field $\overrightarrow{\mathrm{E}}_{\mathrm{s}}(\mathrm{t})$ at the output of the Polarization Beam Combiner (PBC) is expressed as Eq. 5:

$$
\overrightarrow{\mathrm{E}}_{\mathrm{s}}(\mathrm{t})=\sqrt{\frac{\mathrm{P}}{2}} \mathrm{e}^{\mathrm{j}[\omega t+\varphi(t)]}\left\{\varepsilon \mathrm{e}^{\mathrm{j} \gamma} \hat{\mathrm{x}}+\hat{\mathrm{y}}\right\}
$$

where, the modulation function $\gamma \in(0, \pi)$ is for $b_{k} \in(1,0)$ and $\varepsilon \in(1,3,5,7)$ is for $\left\{b_{k 1} \quad b_{k 2}\right\} \in(10,11,01,00)$, respectively. The vector $\mathrm{m}(\mathrm{t})$ is expressed as Eq. 6:

$$
\mathrm{m}(\mathrm{t})=\sum_{\mathrm{k}=-\infty}^{\infty} \mathrm{b}_{\mathrm{k}} \mathrm{rect}_{\mathrm{T}}(\mathrm{t}-\mathrm{kT})
$$

Where:

$$
\begin{aligned}
\mathrm{b}_{\mathrm{k}}=(0,1)= & \text { Transmitted bit } \\
\mathrm{T} & \text { Symbol period and the rectangular pulse } \\
& \text { shaping function } \\
\operatorname{rect}_{\mathrm{T}}(\mathrm{t})= & \text { The rectangular pulse shaping function } \\
& \text { is equal to one for }(\mathrm{t}) \in(0, \mathrm{~T}) \text { and to zero } \\
& \text { elsewhere }
\end{aligned}
$$

Receiver: Figure 2 illustrates the block diagram of the proposed optical coherent heterodyne receiver. The ideal Optical Band-Pass Filter (OBPF) with a narrow bandwidth typically $1 \mathrm{~nm}$ is used to limit the background ambient light. The optical field of the local oscillator $\overrightarrow{\mathrm{E}}_{\mathrm{lo}}(\mathrm{t})$ is linearly polarized at $\pi / 4$ with respect to the receiver reference axes. An Automatic Frequency Control (AFC) is used to compensate for the slowfrequency fluctuations occurring in the Local Oscillator (LO), whose control signal is derived from the intermediate frequency electric signal. Generally, the AFC is a closed-loop circuit acting on the bias current (Betti et al., 1995).

The received optical field and are uncorrelated and can be expressed as Eq. 7a-b:

$$
\begin{aligned}
& \overrightarrow{\mathrm{E}}_{\mathrm{r}}(\mathrm{t})=\sqrt{\frac{\operatorname{Pr}}{2}} \mathrm{e}^{\mathrm{j}[\omega t+\varphi s(t)]}\left\{\varepsilon \mathrm{e}^{\mathrm{j} \gamma} \hat{\mathrm{x}}+\hat{\mathrm{y}}\right\} \\
& \overrightarrow{\mathrm{E}}_{\mathrm{lo}}(\mathrm{t})=\frac{\sqrt{\mathrm{P}_{\mathrm{lo}}}}{2} \mathrm{e}^{\mathrm{j}\left[\omega_{0 \mathrm{o}} t+\phi_{10}(t)\right]}\{\hat{\mathrm{x}}+\hat{\mathrm{y}}\}
\end{aligned}
$$

where, $P_{r}$ and $\varphi_{s}(t)$ are the power and the phase noise of the received optical signal, respectively. Both $P_{r}$ and $\varphi_{\mathrm{s}}(\mathrm{t})$ are time-variant due to the atmospheric turbulence. The parameters $\mathrm{P}_{\mathrm{lo}}, \omega_{\mathrm{lo}}$ and $\varphi_{\mathrm{lo}}(\mathrm{t})$ represent the power, angular frequency and the phase noise of the local oscillator, respectively and $\omega \neq \omega_{\mathrm{lo}}$.

The received optical signal $\overrightarrow{\mathrm{E}}_{\mathrm{r}}(\mathrm{t})$ is split by the PBS into $\hat{x}$ and $\hat{y}$ components which are then mixed with the corresponding optical fields emitted from the local oscillator. Therefore, the decomposed orthogonally polarized components $\overrightarrow{\mathrm{E}}_{\mathrm{x}}(\mathrm{t})$ and $\overrightarrow{\mathrm{E}}_{\mathrm{y}}(\mathrm{t})$ with equal amplitudes are given as Eq. 8a-b:

$$
\begin{aligned}
& \vec{E}_{x}(t)=\left\{\sqrt{\frac{P_{r}}{2}} e^{i\left[\omega t+\gamma+\phi_{s}(t)\right]}+\frac{\sqrt{P_{10}}}{2} e^{j\left[\omega_{0} t+\phi_{10}(t)\right]}\right\} \hat{x} \\
& \vec{E}_{y}(t)=\left\{\sqrt{\frac{P_{r}}{2}} e^{j\left[\omega t+\phi_{s}(t)\right]}+\frac{\sqrt{P_{10}}}{2} e^{j\left[\omega_{00} t+\phi_{10}(t)\right]}\right\} \hat{y}
\end{aligned}
$$

Following optical-to-electrical conversion, the signals $\mathrm{c}_{\mathrm{x}}(\mathrm{t})$ and $\mathrm{c}_{\mathrm{y}}(\mathrm{t})$ at the output of two identical Photo Diodes (PD) are expressed as Eq. 9a-b (Betti et al., 1995):

$$
\begin{aligned}
& c_{x}(t)=R\left\{\begin{array}{l}
\left|\sqrt{\mathrm{P}_{\mathrm{r}} / 2} \varepsilon\right|^{2}+\left|\sqrt{\mathrm{P}_{\mathrm{lo}}} / 2\right|^{2}+ \\
\sqrt{\mathrm{P}_{\mathrm{r}} \mathrm{P}_{\mathrm{lo}} / 2} \varepsilon \cos \left[\omega_{\mathrm{IF}} \mathrm{t}+\gamma+\phi_{\mathrm{IF}}(\mathrm{t})\right]
\end{array}\right\}+\mathrm{n}_{\mathrm{x}}(\mathrm{t}) \\
& \mathrm{c}_{\mathrm{y}}(\mathrm{t})=\mathrm{R}\left\{\begin{array}{l}
\left|\sqrt{\mathrm{P}_{\mathrm{r}} / 2}\right|^{2}+\left|\sqrt{\mathrm{P}_{\mathrm{lo}}} / 2\right|^{2}+ \\
\sqrt{\mathrm{P}_{\mathrm{r}} \mathrm{P}_{\mathrm{lo}} / 2} \cos \left[\omega_{\mathrm{IF}} \mathrm{t}+\phi_{\mathrm{IF}}(\mathrm{t})\right]
\end{array}\right\}+\mathrm{n}_{\mathrm{y}}(\mathrm{t})
\end{aligned}
$$

where, $\mathrm{R}$ represents the photodiode responsively, $\omega_{\mathrm{IF}}=$ $\omega-\omega_{\mathrm{lo}}$ and $\varphi_{\mathrm{IF}}(\mathrm{t})=\varphi_{\mathrm{s}}(\mathrm{t})-\varphi_{\mathrm{lo}}(\mathrm{t})$ are the intermediate angular frequency and the phase noise, respectively. The noise terms $\left(\mathrm{n}_{\mathrm{x}}(\mathrm{t})\right.$ and $\left.\mathrm{n}_{\mathrm{y}}(\mathrm{t})\right)$ represent the background radiation and the thermal noise which are assumed to be statistically independent, stationary Gaussian processes with zero-mean and a variance of $\sigma_{\mathrm{n}}^{2}=\frac{1}{2}$ No, No being the double-sided noise power spectral density consisting of the background radiation noise and the thermal noise. The electrical signals $\mathrm{c}_{\mathrm{x}}(\mathrm{t})$ and $\mathrm{c}_{\mathrm{y}}(\mathrm{t})$ are passed through the ideal Band-Pass Filters (BPF) to reject the constant term and to limit the additive noise. The bandwidth of the BPF is expressed as $B_{b p}=2\left(R_{s}+k_{F} B_{L}\right)$ with the center frequency at $\omega_{I F}$, where $R_{s}$ is the symbol rate and $B_{L}$ is the sum of the transmitting and local oscillator lasers' line width. 


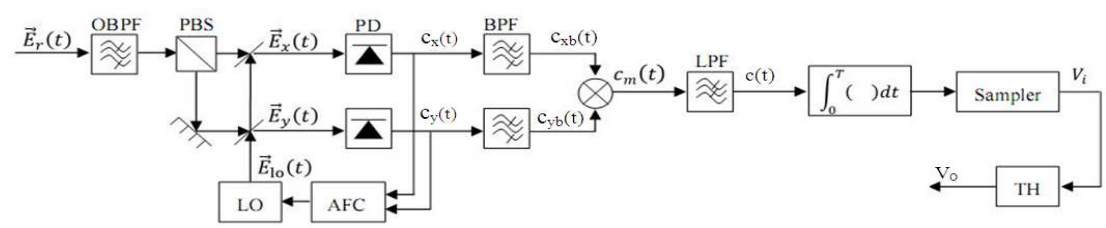

Fig. 2: Block diagram of the optical heterodyne M-POLSK receiver. TH (threshold)

The parameter $\mathrm{k}_{\mathrm{F}}$ must be chosen to transmit the signals undistorted through the filters (Cusani et al., 1992). The $\mathrm{BPF}$ in the lower branch is assumed to have a very narrow bandwidth in order to only pass the carrier signal with negligible distortion. Therefore, the electrical currents at the output of the BPFs are expressed as Eq. 10a-b:

$c_{x b}(t)=\sqrt{R^{2} P_{r} P_{10} / 2} \varepsilon \cos \left[\omega_{I F} t+\gamma+\phi_{I F}(t)\right]+n_{x b}(t)$

$c_{y b}(t)=\sqrt{R^{2} P_{r} P_{l o} / 2} \cos \left[\omega_{I F} t+\varphi_{I F}(t)\right]$

where, $\mathrm{n}_{\mathrm{xb}}(\mathrm{t}) \sim\left(0, \sigma_{\mathrm{n}}{ }^{2}\right)$ is additive Gaussian noise at the output of the BPF. The additive narrowband noise components can be expressed by using the simple trigonometric identities given as Eq. 11 (Proakis, 2001):

$\mathrm{n}_{\mathrm{xb}}(\mathrm{t})=\mathrm{n}_{\mathrm{xb}}^{\mathrm{I}}(\mathrm{t}) \cos \left[\omega_{\text {IF }} \mathrm{t}+\varphi_{\text {IF }}(\mathrm{t})\right]$

$-\mathrm{n}_{\mathrm{xb}}^{\mathrm{Q}}(\mathrm{t}) \sin \left[\omega_{\mathrm{IF}} \mathrm{t}+\varphi_{\mathrm{IF}}(\mathrm{t})\right]$

where, $\mathrm{n}_{\mathrm{xb}}^{\mathrm{I}}(\mathrm{t})$ and $\mathrm{n}_{\mathrm{xb}}^{\mathrm{Q}}(\mathrm{t})$ are the phase and the quadrature components, respectively, which are bandlimited Gaussian with zero mean and a variance of $\sigma_{n}^{2}$. After passing through a Low Pass Filter (LPF), the higher frequency harmonics have been removed. Thus, the baseband current $\mathrm{c}(\mathrm{t})$ is given as Eq. 12:

$$
\begin{aligned}
& \mathrm{c}(\mathrm{t})=\mathrm{c}_{\mathrm{m}}(\mathrm{t}) * \mathrm{~h}_{\mathrm{lp}}(\mathrm{t}) \\
& =\frac{1}{2} \sqrt{\frac{\mathrm{R}^{2} \mathrm{P}_{\mathrm{r}} \mathrm{P}_{\mathrm{lo}}}{2}}\left[\sqrt{\frac{\mathrm{R}^{2} \mathrm{P}_{\mathrm{r}} \mathrm{P}_{\mathrm{lo}}}{2}} \varepsilon \cos (\gamma)+\mathrm{n}_{\mathrm{xb}}^{\mathrm{I}}(\mathrm{t})\right]
\end{aligned}
$$

where, * indicates the convolution operation, $\mathrm{h}_{\mathrm{lp}}(\mathrm{t})$ is the impulse response of the low-pass filter with a bandwidth of $B_{l p}=R_{s}+k_{F} B_{L}$. The electric signal $c(t)$ is integrated over the symbol period $\mathrm{T}$, sampled at time $\mathrm{t}$ $=\mathrm{T}$. Hence, the output of the correlation-type demodulator (Proakis, 2001) is Eq. 13:

$$
\begin{aligned}
& \mathrm{V}_{\mathrm{i}}=\frac{1}{\mathrm{~T}} \int_{0}^{\mathrm{T}} \frac{1}{2} \sqrt{\frac{\mathrm{R}^{2} \mathrm{P}_{\mathrm{r}} \mathrm{P}_{\mathrm{lo}}}{2}}\left[\sqrt{\frac{\mathrm{R}^{2} \mathrm{P}_{\mathrm{r}} \mathrm{P}_{\mathrm{lo}}}{2}} \varepsilon \cos (\gamma)+\mathrm{n}_{\mathrm{xb}}^{\mathrm{I}}(\mathrm{t})\right] \mathrm{dt} \\
& =\frac{1}{2} \sqrt{\frac{\mathrm{R}^{2} \mathrm{P}_{\mathrm{r}} \mathrm{P}_{\mathrm{lo}}}{2 \mathrm{~T}^{2}}}\left[\sqrt{\frac{\mathrm{R}^{2} \mathrm{P}_{\mathrm{r}} \mathrm{P}_{\mathrm{lo}}}{2}} \int_{0}^{\mathrm{T}} \varepsilon \cos (\gamma) \mathrm{dt}+\int_{0}^{\mathrm{T}} \mathrm{n}_{\mathrm{xb}}^{\mathrm{I}}(\mathrm{t}) \mathrm{dt}\right] \\
& =\sqrt{\frac{\mathrm{R}^{2} \mathrm{P}_{\mathrm{r}} \mathrm{P}_{\mathrm{lo}}}{8}}\left[\sqrt{\frac{\mathrm{R}^{2} \mathrm{P}_{\mathrm{r}} \mathrm{P}_{\mathrm{lo}}}{2}} \varepsilon \cos (\gamma)+\mathrm{n}_{\mathrm{xb}}^{\mathrm{I}}\right]
\end{aligned}
$$

The decision rule which maximizes the correlation metrics is applied to determine the average probability of error. It follows that the detector compares the demodulator output $\mathrm{V}_{\mathrm{i}}$ with seven Threshold $(\mathrm{TH})$ levels: $0, \pm \mathrm{R}^{2} \mathrm{PP}_{10} / 2, \pm \mathrm{R}^{2} \mathrm{PP}_{10}, \pm 3 \mathrm{R}^{2} \mathrm{PP}_{10} / 2$. Therefore, a decision is made in favor of the amplitude level closest to $\mathrm{V}_{\mathrm{i}}$.

Error rate analysis: It has been assumed that the data transmission is independent and identically distributed (i.i.d.). For equally probable signals, the decision rule which maximizes the correlation metrics is applied to determine the average probability of error. Compared with the encoded BPOLSK (Tang et al., 2010) the average power for equiprobable coded M-POLSK symbol is increased by a factor of 11 , so that the average power of each symbol in the coded M-POLSK system must be reduced by a factor of 11 . Therefore, the average power per bit is defined by $\mathrm{R}^{2} \mathrm{P}_{\mathrm{r}} \mathrm{P}_{\mathrm{lo}}=\mathrm{P}_{\mathrm{av}} / 22$.

Since an error can only occur in one direction, the BER conditioned on the received optical power before the decoder is expressed as Eq. 14:

$$
\begin{aligned}
& P_{c e}=\frac{1}{4} \frac{1}{\sqrt{\pi N o}}\left[\begin{array}{l}
\int_{-\infty}^{0} \mathrm{e}^{-\frac{\left(x-\sqrt{R^{2} P_{r} P_{\mathrm{P}_{0}} / 2}\right)^{2}}{\mathrm{No}}} \mathrm{dx}+ \\
\int_{2 \sqrt{R^{2} \mathrm{P}_{\mathrm{r}} \mathrm{P}_{\mathrm{to}} / 2}}^{\infty} \mathrm{e}^{\frac{\left(x-\sqrt{R^{2} P_{\mathrm{r}} \mathrm{P}_{\mathrm{to}} / 2}\right)^{2}}{\mathrm{No}}} \mathrm{dx}
\end{array}\right.
\end{aligned}
$$

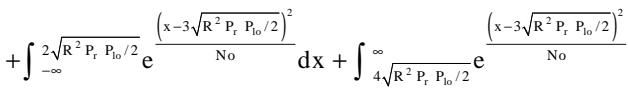

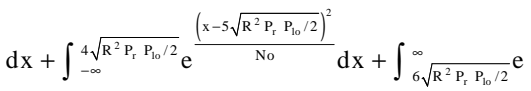

$$
\begin{aligned}
& \left.\frac{\left(x-5 \sqrt{R^{2} P_{r} P_{\mathrm{P}_{10}} / 2}\right)^{2}}{N_{0}} \mathrm{dx}+\int_{-\infty}^{6 \sqrt{R^{2} P_{\mathrm{r}} \mathrm{P}_{\mathrm{lo}} / 2}} \mathrm{e} \frac{\left(x-7 \sqrt{\mathrm{R}^{2} \mathrm{P}_{\mathrm{r}} \mathrm{P}_{\mathrm{lo}} / 2}\right)^{2}}{\mathrm{No}} \mathrm{dx}\right]
\end{aligned}
$$

In addition, simplifying (14) and substituting the average power per bit, the BER expression for the coded M-POLSK before the decoder is expressed as Eq. 15:

$$
\begin{aligned}
& \mathrm{P}_{\mathrm{ce}}=\frac{7}{4} \frac{1}{\sqrt{2 \pi}} \frac{\int^{\frac{\mathrm{R}_{\mathrm{P}} \mathrm{P}_{\mathrm{b}}}{\mathrm{N}_{\mathrm{o}}}}}{\infty} \mathrm{e}^{-\frac{\mathrm{x}^{2}}{2}} \mathrm{dx}=\frac{7}{4} \mathrm{Q}\left(\sqrt{\frac{\mathrm{R}^{2} \mathrm{P}_{\mathrm{r}} \mathrm{P}_{\mathrm{lo}}}{\mathrm{N}_{0}}}\right) \\
& =\frac{7}{4} \mathrm{Q}\left(\sqrt{\frac{\mathrm{P}_{\mathrm{av}}}{22 \mathrm{~N}_{0}}}\right)=\frac{7}{4} \mathrm{Q}\left(\sqrt{\frac{\mathrm{r}}{22}}\right)
\end{aligned}
$$

where, $\mathrm{Q}($.$) is the Gaussian- \mathrm{Q}$ function and $\mathrm{r}=\mathrm{P}_{\mathrm{av}} / \mathrm{No}$ is the electrical Signal to Noise Ratio (SNR) at the input of the demodulator. 
The PEP is the basic method for the union bounds calculation, which is used as the main criterion for code design (Sandalidis, 2011; Simon and Alouini, 2005). Under the assumption of the maximum likelihood soft decoding with perfect Channel State Information (CSI), the conditional PEP subject to the fading coefficients $\mathrm{I}=\mathrm{I}_{1}, \mathrm{I}_{2}, \ldots, \mathrm{I}_{\mathrm{k}}$ is expressed as Eq: 16 (Zhu and Kahn, 2003; Uysal et al., 2006):

$\mathrm{P}(\mathrm{X}, \hat{\mathrm{X}} \mid \mathrm{I})=\frac{7}{4} \mathrm{Q}\left(\sqrt{\frac{\mathrm{r}}{22} \sum_{\mathrm{k} \in \Omega} \mathrm{I}_{\mathrm{k}}^{2}}\right)$

where, $\tilde{\mathrm{x}}=\left(\tilde{\mathrm{x}}_{1}, \ldots \tilde{\mathrm{x}}_{\mathrm{n}}\right)$ and $\mathrm{x}=\left(\mathrm{x}_{1}, \ldots \mathrm{x}_{\mathrm{n}}\right)$ stand for the choosing coded sequence and the transmission sequence, respectively and $\Omega$ is the set of bit intervals' locations where $\mathbf{X}$ and $\tilde{\mathbf{x}}$ differ from each other. The alternative function form for the Gaussian-Q function is Eq. 17 (Sandalidis, 2011; Simon and Alouini, 2005):

$\mathrm{Q}(\mathrm{x})=\frac{1}{\pi} \int_{0}^{\pi / 2} \exp \left(-\frac{\mathrm{x}^{2}}{2 \sin ^{2} \theta}\right) \mathrm{d} \theta$

Substituting Eq. 16 into Eq. 17 and considering the received signal light subjected to fading, Eq. 16 becomes Eq. 18:

$\mathrm{P}(\mathrm{X}, \hat{\mathrm{X}} \mid \mathrm{I})=\frac{1}{\pi} \int_{0}^{\pi / 2} \frac{7}{4} \Pi_{\mathrm{k} \in \Omega} \exp \left(-\frac{\mathrm{r}}{44} \frac{\mathrm{I}_{\mathrm{k}}^{2}}{\sin ^{2} \theta}\right) \mathrm{d} \theta$

We need to take Eq. 18 as regards $I_{k}$ to obtain the unconditional PEP. Assuming perfect interleaving, the independency among fading coefficients $I_{k}$ can be exploited and the unconditional PEP is wrote as Eq. 19:

$$
\begin{aligned}
& \mathrm{P}(\mathrm{X}, \hat{\mathrm{X}})=\frac{7}{4} \frac{1}{\pi} \int_{0}^{\pi / 2} \Pi_{\mathrm{k} \in \Omega} \mathrm{E}_{\mathrm{I}_{\mathrm{k}}}\left[\exp \left(-\frac{\mathrm{r}}{44} \frac{\mathrm{I}_{\mathrm{k}}^{2}}{\sin ^{2} \theta}\right)\right] \mathrm{d} \theta \\
& =\frac{7}{4} \frac{1}{\pi} \int_{0}^{\pi / 2}\left[\int_{0}^{\infty} \exp \left(-\frac{\mathrm{r}}{44} \frac{\mathrm{I}^{2}}{\sin ^{2} \theta}\right) \mathrm{p}(\mathrm{I}) \mathrm{dI}\right]^{|\Omega|} \mathrm{d} \theta
\end{aligned}
$$

Where:

$\mathrm{E}()=$. The expectation operation

$|\Omega|=$ The cardinality of $\Omega$ corresponding to the length of error event

Note that Eq. 19 has no closed form solution. The unconditional PEP Eq. 19 is derived for uncoded MPOLSK modulated FSO communication systems operating over gamma-gamma turbulence channel. The unconditional PEP expression is the tool for the derivation of upper bounds on the error probability of the coded M-POLSK communication system.

Maximum likelihood sequence detection: In order to reduce the effects of the turbulence induced fading on the received optical signal, a convolution coded M-
POLSK-FSO communication system has been considered. The union upper bound on the average BER with uniform error probability codes can be found as Eq. 20 (Sandalidis, 2011; Simon and Alouini, 2005):

$$
\mathrm{P}_{\mathrm{b}} \leq \frac{7}{4} \frac{1}{\pi} \int_{0}^{\pi / 2}\left[\left.\frac{1}{\mathrm{n}} \frac{\partial}{\mathrm{n} \partial \mathrm{N}} \mathrm{T}(\mathrm{D}(\theta), \mathrm{N})\right|_{\mathrm{N}=1}\right] \mathrm{d} \theta
$$

where, $\mathrm{N}$ is an indicator taking into account the number of bits in error, $\mathrm{n}$ is the number of information bits per transmission, the transfer function $T(D(\theta), N)$ is in conjunction with the particular state diagram of a coded modulation and $\mathrm{D}(\theta)$ depends on the derived PEP. Here we have applied a convolutional code with the rate of $1 / 3$ and the constraint length of 3 , as illustrated in Fig. 8.2-2 of (Proakis, 2001). The function generators of the convolutional encoder are given as and $\mathrm{g}_{1}=(100), \mathrm{g}_{2}=$ (101) and $g_{3}=(111)$. The transfer function is expressed as Eq. 21:

$\mathrm{T}(\mathrm{D}(\theta), \mathrm{N})=\frac{\mathrm{D}^{6}(\theta) \mathrm{N}}{1-2 \mathrm{ND}^{2}(\theta)}$

The BER is thus obtained as Eq. 22:

$\mathrm{P}_{\mathrm{b}} \leq \frac{7}{4} \frac{1}{\pi} \int_{0}^{\pi / 2} \frac{\mathrm{D}^{6}(\theta)}{\left(1-2 \mathrm{D}^{2}(\theta)\right)^{2}} \mathrm{~d} \theta$

$\mathrm{D}(\theta)$ is defined based on the underlying PEP expression. In this study, using the integrand of PEP expression given by Eq. 20, the approximation $\mathrm{D}(\theta)$ formula for the channels under consideration is Eq. 23:

$$
\mathrm{D}(\theta)=\int_{0}^{\infty} \exp \left(-\frac{\mathrm{r}}{44} \frac{\mathrm{I}^{2}}{\sin ^{2} \theta}\right) \mathrm{p}(\mathrm{I}) \mathrm{dI}
$$

Spatial diversity techniques: Employing multiple photo-detectors can mitigate the turbulence induced fading in the received signal, thus leading to further improvement in the error performance. To avoid any correlation in the received irradiance the detectors are sufficiently spaced as shown in Fig. 3 .

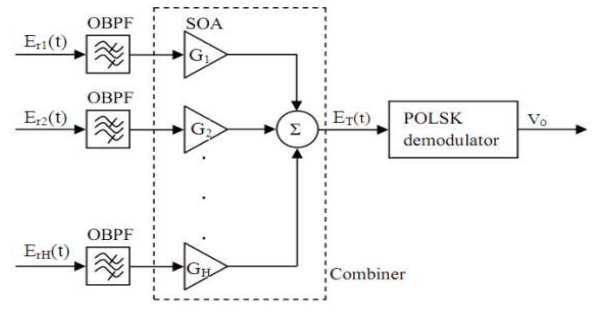

Fig. 3: Spatial diversity receiver with H-PDs PhotoDetector (PD); Semiconductor Optical Amplifier (SOA) 
Since the transverse correlation size $\rho_{0}$ of the laser radiation operating over the atmospheric turbulence channel is nearly a few centimeters, the parameter $\rho_{0}$ can be assumed to be greater than the spacing between the detectors (Popoola and Ghassemlooy, 2009). Since the spacing between the PDs is much shorter than the wireless link range, the difference in the propagation delay across the receiver array would be negligible (Popoola and Ghassemlooy, 2009). Note that the received optical power is assumed to be constant and time invariant during $\mathrm{T}<\tau_{0}$, where the coherence time $\tau_{0}$ of the atmospheric fluctuation is in the order of milliseconds (Shin and Chan, 2004).

The received signal from each branch is scaled by a gain factor $\left\{\mathrm{G}_{\mathrm{i}}\right\}_{\mathrm{i}=1}^{\mathrm{H}}$. The output of the combiner is the sum of the weighted and co-phased signals as illustrated in Fig. 3. Each receiver aperture size of the H-photo detector is $(1 / \mathrm{H})^{\text {th }}$ of the aperture area of the single receiver. Accordingly, the background noise variance on each branch is proportional to the receiver aperture area, which is reduced by a factor of $\mathrm{H}$. Whereas the thermal noise on each branch is not affected. It follows that the total noise variance at the combiner output is $\sigma_{\mathrm{sp}}^{2}=\mathrm{H} \sigma_{\text {Thermal }}^{2}+\sigma_{\mathrm{Bg}}^{2}$. The background noise is not considered here because the ideal OBPF with a narrow FOV is applied at the receiver. Hence, $\sigma_{\mathrm{Bg}}^{2}<H \sigma_{\text {Thermal }}^{2}$ and $\sigma_{\mathrm{sp}}^{2} \cong \sigma_{\text {Thermal }}^{2}$ the variance of the overall noise with a zero mean becomes as Eq. 24:

$\mathrm{N}_{\mathrm{T}}=\sum_{\mathrm{i}=1}^{\mathrm{H}} \mathrm{G}_{\mathrm{i}}^{2} \mathrm{No}, \mathrm{i}=1,2 \ldots . \mathrm{H}$

The total received optical power during the symbol duration at the output of the combiner is given as Eq. 25 :

$\overrightarrow{\mathrm{E}}_{\mathrm{T}}(\mathrm{t})=\frac{1}{\mathrm{H}} \sum_{\mathrm{i}=1}^{\mathrm{H}} \sqrt{\frac{\mathrm{G}_{\mathrm{i}}^{2} \mathrm{P}_{\mathrm{ri}}}{2}} \mathrm{e}^{\mathrm{j}\left[\omega t+\phi_{\mathrm{s}}(t)\right]}\left\{\varepsilon \mathrm{e}^{\mathrm{j} \mathrm{x}} \hat{\mathrm{x}}+\hat{\mathrm{y}}\right\} \cdot$

The optimum post detection electrical SNR $r_{\mathrm{T}}$ at the M-POLSK demodulator input becomes as Eq. 26:

$\mathrm{r}_{\mathrm{T}}=\frac{\sum_{\mathrm{i}=1}^{\mathrm{H}} \mathrm{G}_{\mathrm{i}}^{2} \mathrm{P}_{\mathrm{avi}}}{\mathrm{H}^{2} \sum_{\mathrm{i}=1}^{\mathrm{H}} \mathrm{G}_{\mathrm{i}}^{2} \mathrm{No}}$

where, $\quad \overrightarrow{\mathrm{I}}^{2}=\left(\sum_{\mathrm{i}=1}^{\mathrm{H}} \mathrm{I}_{i}\right)^{2}$ and $\mathrm{p}(\overrightarrow{\mathrm{I}})=\prod_{\mathrm{i}=1}^{\mathrm{H}} \mathrm{p}\left(\mathrm{I}_{\mathrm{i}}\right)$ represents the joint PDF of the uncorrelated irradiance. Hypothesises are taken that all the received optical power is independent and all obey gamma-gamma distribution.
Therefore, $\mathrm{p}(\overrightarrow{\mathrm{I}})$, being the joint PDF for $\mathrm{H}$ photodetector receiving uncorrelated signals, is expressed as Eq. 27:

$$
\begin{aligned}
& \mathrm{p}(\overrightarrow{\mathrm{I}})=\int \ldots \iint \mathrm{p}\left(\mathrm{I}_{1}\right) \ldots \mathrm{p}\left(\mathrm{I}_{\mathrm{H}}\right) \mathrm{dI}_{1} \ldots \mathrm{dI}_{\mathrm{H}} \\
& =\prod_{\mathrm{i}=1}^{\mathrm{H}} \int \mathrm{p}\left(\mathrm{I}_{\mathrm{i}}\right) \mathrm{dI}_{\mathrm{i}}
\end{aligned}
$$

The system performance of the coded M-POLSKbased FSO communication system operating over gamma-gamma turbulence channel can thus be evaluated from Eq. 2, 22 and 27 using numerical integration since the resulting expression has no closed form. In determining $\mathrm{r}_{\mathrm{T}}$, only MRC spatial diversity techniques are considered in this study. As the MRC linear combiner results in a maximum-likelihood receiver structure (Simon and Alouini, 2005), it is optimal regardless of the fading statistics.

The received power level on every branch has to be estimated prior for the coherent summation. The gain factor $\left\{\mathrm{G}_{\mathrm{i}}\right\}_{\mathrm{i}=1}^{\mathrm{H}}$ is proportional to the received optical power. Applying the Cauchy inequality (Gradshtein et al., 2007), $\sum_{\mathrm{i}=1}^{\mathrm{H}} \mathrm{G}_{\mathrm{i}}^{2} \mathrm{P}_{\text {avi }} \leq\left(\sum_{\mathrm{i}=1}^{\mathrm{H}} \mathrm{G}_{\mathrm{i}}^{2}\right)\left(\sum_{\mathrm{i}=1}^{\mathrm{H}} \mathrm{P}_{\text {avi }}\right)$, the is derived as Eq. 28:

$$
\mathrm{r}_{\mathrm{MRC}} \leq \frac{\sum_{\mathrm{i}=1}^{\mathrm{H}} \mathrm{G}_{\mathrm{i}}^{2} \mathrm{No}\left(\sum_{\mathrm{i}=1}^{\mathrm{H}} \mathrm{P}_{\text {avi }} / \mathrm{No}\right)}{\mathrm{H}^{2} \sum_{\mathrm{i}=1}^{\mathrm{H}}\left(\mathrm{G}_{\mathrm{i}}^{2} \mathrm{No}\right)}=\frac{1}{\mathrm{H}^{2}}\left(\sum_{\mathrm{i}=1}^{\mathrm{H}} \frac{\mathrm{P}_{\mathrm{avi}}}{\mathrm{No}}\right)
$$

Therefore, the unconditional BER is derived as Eq. 29:

$\mathrm{P}_{\mathrm{MRC}}=\int_{0}^{\infty} \frac{7}{4} \mathrm{Q}\left(\sqrt{\frac{\mathrm{r}_{\mathrm{MRC}} \Pi_{\mathrm{k} \Omega \Omega} \mathrm{I}_{\mathrm{k}}^{2}}{22}}\right) \mathrm{p}(\overrightarrow{\mathrm{I}}) \mathrm{d} \overrightarrow{\mathrm{I}}$

\section{RESULTS}

To evaluate the system performances of the proposed coded M-POLSK based FSO communication link, the error probability of the system employing the coherent BPOLSK, BPSK and DPSK schemes are illustrated. For the purpose of like-to-like comparison, the average optical signal power $E\left[R_{\mathrm{r}} \mathrm{P}_{\mathrm{lo}}\right]$ is normalized to unity and the electrical SNRs are made equal for different modulation schemes. Furthermore, the channel turbulence under consideration is from weak to strong regimes. The values of $\alpha$ and $\beta$ at any given regimes can be calculated with the corresponding value of $\sigma_{1}^{2}$ using Eq. 3. The values of all the parameters used in calculations are illustrated in Table 1. 
Table 1: Simulation parameters with respect to weak, moderate and strong turbulence regimes

\begin{tabular}{llllll}
\hline $\begin{array}{l}\text { Wavelength } \\
\text { Refractive index }\end{array}$ & $\begin{array}{l}\lambda=1550 \mathrm{~nm} \\
\mathrm{c}_{\mathrm{n}}{ }^{2}=1.7 \times 10^{-14}\end{array}$ \\
Diameter of the receiver & $\mathrm{D}<<\mathrm{L}$ and $\mathrm{d}=0$ \\
\hline Link length & $\mathrm{L}=3 \mathrm{~km}$ & $\sigma_{1}{ }^{2}=1.03$ & $\alpha=2.902$ & $\beta=2.51 \quad$ weak \\
& $\mathrm{L}=4 \mathrm{~km}$ & $\sigma_{1}{ }^{2}=1.75$ & $\alpha=2.296$ & $\beta=1.822$ & Moderate \\
& $\mathrm{L}=6 \mathrm{~km}$ & $\sigma_{1}{ }^{2}=3.67$ & $\alpha=2.064$ & $\beta=1.342$ & Strong \\
\hline
\end{tabular}

The BER expressions for BPOLSK with and without turbulence are given as Eq. 30a-b (Tang et al., 2010):

$$
\begin{aligned}
& \mathrm{P}_{\mathrm{BPOLSK}}=\mathrm{Q}\left(\sqrt{\mathrm{r} \sum_{\mathrm{k} \in \Omega} \mathrm{I}_{\mathrm{k}}^{2}}\right) \\
& \mathrm{P}_{\mathrm{BPOLSK}}^{\prime}=\int_{0}^{\infty} \mathrm{Q}\left(\sqrt{\sum_{\mathrm{k} \in \Omega} \mathrm{I}_{\mathrm{k}}^{2}}\right) \mathrm{p}(\mathrm{I}) \mathrm{dI}
\end{aligned}
$$

Following the approach adopted in (Zhu and Kahn, 2002), the error probabilities of the DPSK modulated coherent system in the absence and presence of the turbulence channel is given as Eq. 31a-b:

$$
\begin{aligned}
& \mathrm{P}_{\text {DPSK }}=\operatorname{erfc}\left(\sqrt{\mathrm{r} \sum_{\mathrm{k} \in \Omega} \mathrm{I}_{\mathrm{k}}^{2}}\right)\left[1-\frac{1}{2} \operatorname{erfc}\left(\sqrt{\mathrm{r} \sum_{\mathrm{k} \in \Omega} \mathrm{I}_{\mathrm{k}}^{2}}\right)\right] \\
& \mathrm{P}_{\mathrm{DPSK}}^{\prime}=\int_{0}^{\infty} \operatorname{erfc}\left(\sqrt{\mathrm{r} \sum_{\mathrm{k} \in \Omega} \mathrm{I}_{\mathrm{k}}^{2}}\right)\left[1-\frac{1}{2} \operatorname{erfc}\left(\sqrt{\mathrm{r} \sum_{\mathrm{k} \in \Omega} \mathrm{I}_{\mathrm{k}}^{2}}\right)\right] \mathrm{p}(\mathrm{I}) \mathrm{dI}
\end{aligned}
$$

The conditional BER expressions for the coherent BPSK modulation technique without and with the phase tracking errors are given as Eq. 32a-b (Betti et al., 1995):

$$
\begin{aligned}
& \mathrm{P}_{\mathrm{BPSK}}=\mathrm{Q}\left(\sqrt{2 \mathrm{r} \sum_{\mathrm{k} \in \Omega} \mathrm{I}_{\mathrm{k}}^{2}}\right) \\
& \mathrm{P}_{\mathrm{BPSK}}^{\prime}=\int_{0}^{2 \pi} \mathrm{Q}\left(\sqrt{2 \mathrm{r} \sum_{\mathrm{k} \in \Omega} \mathrm{I}_{\mathrm{k}}^{2}} \cos (\Delta \varphi)\right) \frac{\mathrm{e}^{\mathrm{r} \cos (\Delta \varphi)}}{2 \pi \mathrm{I}_{0}\left(\mathrm{r}_{1}\right)} \mathrm{d} \Delta \varphi
\end{aligned}
$$

where, $\Delta \emptyset$ denotes the phase tracking error due to the PLL circuit and $r_{1}=1 / \sigma_{\Delta \emptyset}^{2}$ with the phase tracking error variance $\sigma_{\Delta \emptyset}$. Therefore, the theoretical unconditional BER for the BPSK scheme in the gamma-gamma turbulence channel is derived as Eq. 32c:

$$
\mathrm{P}_{\mathrm{BPSK}}^{\mathrm{\prime}}\left(\mathrm{P}_{\mathrm{r}}\right)=\int_{0}^{\infty} \int_{0}^{2 \pi} \mathrm{Q}\left(\sqrt{2 \mathrm{r} \sum_{\mathrm{k} \in \Omega} \mathrm{I}_{k}^{2}} \cos (\Delta \varphi) \frac{\mathrm{e}^{\mathrm{r}_{\mathrm{I}} \cos (\Delta \varphi)}}{2 \pi \mathrm{I}_{0}\left(\mathrm{r}_{1}\right)} \mathrm{p}(\mathrm{I}) \mathrm{d} \Delta \varphi \mathrm{dI}\right.
$$

\section{DISCUSSION}

The error performances of coherent BPOLSK, DPSK and BPSK schemes can be predicted for any given value of SNR across all turbulence regimes using Eq. 30, 31 and 32 as shown in Fig. 4.

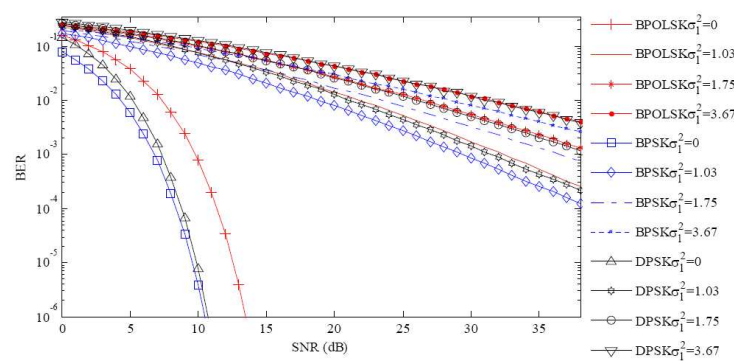

Fig. 4: BER performances against the normalized SNR $\mathrm{E}[\mathrm{RPrPlo}]=1$ for different coherent modulation schemes across all turbulence regimes

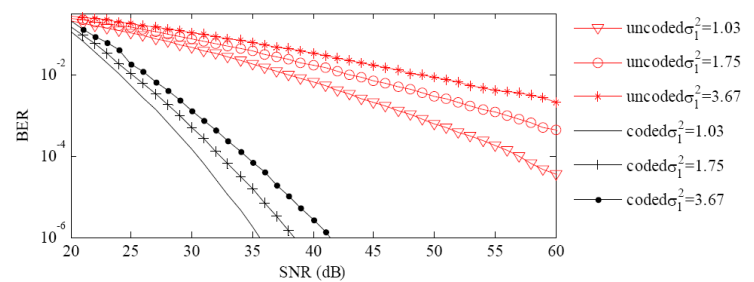

Fig. 5: The comparisons of BER performances between uncoded and coded M-POLSK Eq. 19 and $\mathrm{Pb}$ Eq. 22 against the normalized electrical SNR $\mathrm{E}[\mathrm{RPrPlo}]=1$ for various turbulence levels

The SNR requirements to achieve a BER of $10^{-6}$ without turbulence are $\sim 10.5, \sim 10.7$ and $\sim 13.5 \mathrm{~dB}$ for BPSK (without phase tracking errors), DPSK and BPOLSK, respectively. The BPSK-FSO system offers the best performance in terms of error probability followed by the DPSK scheme for all turbulence scenarios. The BPOLSK provides the worst performance in a turbulence channel. This is due to the fact that only half of the emitted optical power is allocated for the information embedded optical carrier whereas the other half is used for the transmission of the optical reference carrier. It follows that the BPOLSK scheme requires no carrier recovery circuit at the receiver and both the carrier and information signals suffer the same phase noise. However, BPSK and DPSK incur penalties due to the phase tracking errors.

The BER results in Fig. 5 are computed based on Eqs. 19 and 22 to allow comparisons of the performances of the uncoded and coded M-POLSK schemes as well as the uncoded BPOLSK scheme (Fig. 4 ) in the turbulence channel. The performance of the convolutional coded M-POLSK is made evident from the figure. The coded M-POLSK and uncoded BPOLSK schemes have the same spectral efficiency of 1bit/symbol. Compared with BPOLSK scheme, the BER performances deteriorate fast for uncoded $\mathrm{M}$ POLSK scheme for all FSO scenarios under investigation. 
Am. J. Engg. \& Applied Sci., 4 (4): 520-530, 2011

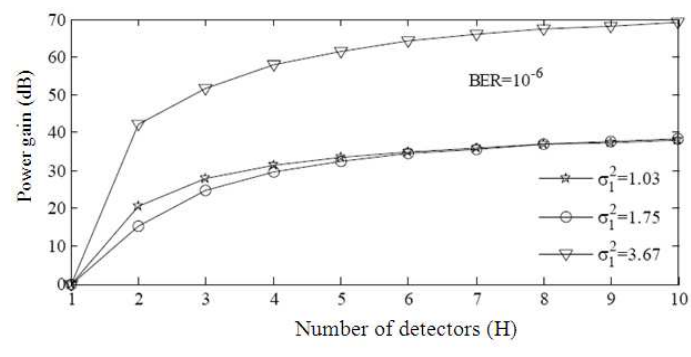

Fig. 6: The power gain for the M-POLSK schme employing MRC technique to achieve a BER of $10^{-6}$ gainst the number of detectors with the normalized electrical SNR E $\left[\mathrm{RP}_{\mathrm{r}} \mathrm{P}_{\mathrm{lo}}\right]=1$ under all turbulence scenarios fromweak to strong regimes

However, as revealed out by the results under all turbulence assumptions, increasing SNR results in a relatively smaller change in the slope of the BER curves for uncoded M-POLSK. The reduction in the SNR for coded M-POLSK is achieved by the use of convolutional code in mitigating the effect of turbulence induced irradiance fluctuation. For example, with the SNR of $30 \mathrm{~dB}$, the BERs for uncoded and coded M-POLSK are 0.047 and $1.4 \times 10^{-4}$ respectively in weak turbulence regime. With the same SNR in the moderate regime, the BER drops from 0.08 to $5.13 \times 10^{-4}$ for the uncoded and coded M-POLSK, respectively.

The BER based on the derived PEP yields a very good performance, which is achieved without the need for the bandwidth expansion. It is not practical and even not feasible for many applications to increase the power margin in the link budget in order to eliminate the deep fades observed under turbulence. This motivates the employment of powerful scintillation-mitigation techniques, such as coding and/or diversity techniques.

Using Eq. 29 the power gain $\left(\mathrm{SNR}_{\sigma \mathrm{\sigma l}, \mathrm{H}}-\mathrm{SNR}_{\sigma \mathrm{l}, 1}\right)$ to achieve a BER of $10^{-6}$ for the MRC technique is depicted in Fig. 6. The MRC technique $(\mathrm{H}=4)$ outperforms the single receiver by $\sim 31.4, \sim 29.5$ and $\sim 57.9 \mathrm{~dB}$ respectively in weak, moderate and strong turbulence regimes, respectively. The power gain is less in the moderate turbulence regime than in the weak regime. This is because of the deep fades resulting from a loss of spatial coherence of the laser radiation. The power gain reaches up to $\sim 69 \mathrm{~dB}$ when ten detectors are used in strong turbulence regime. The power gain is higher in strong fading conditions since adding more detectors will efficiently reduce the chance of a catastrophic fading. Another observation from Fig. 6 is that as the number of detectors $(\mathrm{H})$ increases, the power gain starts flatten out. For example, increasing the number of detectors from four to five only achieves $\sim 2$, $\sim 3$ and $\sim 3.6 \mathrm{~dB}$ more in power gain from weak to strong turbulence regimes, respectively. Thus, the optimum number of the detectors is $2 \leq \mathrm{H} \leq 4$. As a consequence, the power gain is achieved with the rise in system complexity and cost.

\section{CONCLUSION}

This study has outlined the theoretical analysis of a coherent M-POLSK modulated FSO communication system operating over the gamma-gamma turbulence channel. To mitigate the turbulence induced fading the convolution coding and the spatial diversity with the MRC technique have been applied. The upper BER bound based on the derived PEP has been obtained using the transfer function. The BER yields a very good performance, which is achieved without the need for increasing the SNR. For example, with a SNR of $30 \mathrm{~dB}$, the BERs for uncoded and coded M-POLSK schemes are 0.047 and $1.4 \times 10^{-4}$ respectively, in the weak turbulence regime. Around $69 \mathrm{~dB}$ power gain is achievable when ten detectors are used in strong turbulence regime. The spatial diversity with MRC technique $(\mathrm{H}=4)$ outperforms the uncoded M-POLSK employing the single receiver by $\sim 31.4, \sim 29.5$ and $\sim 57.9 \mathrm{~dB}$ respectively in weak, moderate and strong turbulence regimes. We also have shown that the spatial diversity offers increased link margin as the scintillation level rises. The performances of BPOLSK, BPSK and DPSK based FSO systems have been evaluated across all turbulence regimes. The BPSK without phase tracking error outperforms BPOLSK in terms of the SNR to achieve the same BER performance for a range of turbulence regimes. However, the BPSK scheme suffers the penalties due to the phase tracking errors. The performance degradation increases with the phase error variances.

\section{ACKNOWLEDGMENT}

Study is supported by the EU Cost Action, IC802 program.

\section{REFERENCES}

Abtahi, M., P. Lemieux, W. Mathlouthi and L.A. Rusch, 2006. Suppression of turbulence-induced scintillation in free-space optical communication systems using saturated optical amplifiers. J. Lightwave Technol., 24: 4966-4973. DOI: 10.1109/JLT.2006.884561 
Al-Habash, M.A., L.C. Andrews and R.L. Phillips, 2001. Mathematical model for the irradiance probability density function of a laser beam propagating through turbulent media. Opt. Eng., 40: $1554-1562$.

Andrews, L.C., R.L. Philips and C.Y. Hopen, 2001. Laser Beam Scintillation with Applications. 1st Edn., SPIE Press, Bellingham, ISBN: 10: 0819441031, pp: 375.

Arnon, S., 2003. Effects of atmospheric turbulence and building sway on optical wireless-communication systems. Optics Lett., 28: 129-131. DOI: 10.1364/OL.28.000129

Betti, S., G.D. Marchis and E. Iannone, 1995. Coherent Optical Communications Systems. 1st Edn. John Wiley, New York, ISBN: 10: 0471575127, pp: 539.

Cusani, R., E. Iannone, A.M. Salonico and M. Todaro, 1992. An efficient multilevel coherent optical system: M-4Q-QAM. J. Lightwave Technol., 10: 777-786. DOI: $10.1109 / 50.143078$

Goodman, J.W., 1985. Statistical optics. 1st Edn., Wiley-Interscience, New York, ISBN: 10: 0471015024, pp: 550.

Gradshtein, I.S., A. Jeffrey and D. Zwillinger, 2007. Table of Integrals, Series and Products. 7th Edn., Academic Press, London, ISBN: 0123736374, pp: 1171.

Kamalakis, T., T. Sphicopoulos, S.S. Muhammad and E. Leitgeb, 2006. Estimation of the power scintillation probability density function in freespace optical links by use of multicanonical Monte Carlo sampling. Optics Lett., 31: 3077-3079. DOI: 10.1364/OL.31.003077

Karp, S., 1988. Optical Channels: Fibers, Clouds, Water and the Atmosphere. 1st Edn., Plenum Press, New York, ISBN: 10: 0306426544, pp: 405.

Khalighi, M.A., N. Schwartz, N. Aitamer and S. Bourennane, 2009. Fading reduction by aperture averaging and spatial diversity in optical wireless systems. J. Opt. Commun. Netw., 1: 580-593. DOI: 10.1364/JOCN.1.000580

Osche, G.R., 2002. Optical Detection Theory For Laser Applications. 1st Edn., Wiley-Interscience, Hoboken, New Jersey, ISBN: 0471224111, pp: 412.

Popoola, W.O. and Z. Ghassemlooy, 2009. BPSK subcarrier intensity modulated free-space optical communications in atmospheric turbulence. J. Lightwave Technol., 27: 967-973. DOI: 10.1109/JLT.2008.2004950

Popoola, W.O., Z. Ghassemlooy, J.I.H. Allen, E. Leitgeb and S. Gao, 2008. Free-space optical communication employing subcarrier modulation and spatial diversity in atmospheric turbulence channel. IET Optoelect., 2: 16-23. DOI: 10.1049/iet-opt:20070030
Proakis, J.G., 2001. Digital Communications. 4th Edn., McGraw-Hill, Boston, ISBN: 10: 0072321113, pp: 1002.

Ramirez-Iniguez, R., S.M. Idrus and Z. Sun, 2008. Optical Wireless Communications: IR for Wireless Connectivity. 1st Edn., CRC Press, London, ISBN: 10: 0849372097, pp: 344.

Sandalidis, H.G., 2011. Coded free-space optical links over strong turbulence and misalignment fading channels. IEEE Trans. Commun., 59: 669-674. DOI: 10.1109/TCOMM.2011.121410.090318

Shin, E.J. and V.W.S. Chan, 2004. Part 1: Optical communication over the clear turbulent atmospheric channel using diversity. IEEE J. Select. Areas Commun., 22: 1896-1906. DOI: 10.1109/JSAC.2004.835751

Simon, M.K. and M.S. Alouini, 2005. Digital Communication over Fading Channels. 2nd Edn., John Wiley and Sons, Hoboken, New Jersey, ISBN: 9780471649533, pp: 900.

Sugianto, T. and C.C. Davis, 2006. Performance of free space optical communication systems using polarization shift keying modulation. Proc. SPIE Int. Soc. Opt. Eng., 6304: 63040V-63040V.

Tang, X., Z. Ghassemlooy, S. Rajbhandari, W.O. Popoola and C.G. Lee, 2011. Coherent optical binary polarisation shift keying heterodyne system in the free-space optical turbulence channel. IET Microw. Antennas Propag., 5: 1031-1038. DOI: 10.1049/iet-map.2010.0221

Tang, X., Z. Ghassemlooy, S. Rajbhandari, W.O. Popoola and C.G. Lee et al., 2010. Free-space optical communication employing polarization shift keying coherent modulation in atmospheric turbulence channel. Proceedings of the 7th International Symposium on Communication Systems, Networks and Digital Signal Processing, Jul. 21-23, IEEE Xplore Press, Newcastle Upon Tyne, UK, pp: 615-620.

Uysal, M., J. Li and M. Yu, 2006. Error rate performance analysis of coded free-space optical links over gamma-gamma atmospheric turbulence channels. IEEE Trans. Wireless Commun., 5: 1229-1233. DOI: 10.1109/TWC.2006.1638639

Weyrauch, T. and M.A. Vorontsov, 2004. Free-space laser communications with adaptive optics: Atmospheric compensation experiments. J. Opti. Fiber Commun. Res., 1: 355-379. DOI: 10.1007/s10297-005-0033-5 
Am. J. Engg. \& Applied Sci., 4 (4): 520-530, 2011

Zhu, X. and J.M. Kahn, 2002. Free-space optical communication through atmospheric turbulence channels. IEEE Trans. Commun., 50: 1293-1300. DOI: 10.1109/TCOMM.2002.800829

Zhu, X. and J.M. Kahn, 2003. Performance bounds for coded free-space optical communications through atmospheric turbulence channels. IEEE Trans. Commun., 51: 1233-1239. DOI: 10.1109/TCOMM.2003.815052
Zhu, X. and J.M. Kahn, 2001. Pairwise codeword error probability for coded free-space optical communication through atmospheric turbulence channels. Proceedings of the IEEE International Conference on Communications, Jun. 11-14, IEEE Xplore Press, Helsinki, Finland, pp: 161-164. DOI: 10.1109/ICC.2001.936294 